\title{
Ingestion volontaire et préférences alimentaires chez des moutons Djallonké nourris avec des graminées et des légumineuses fourragères tropicales cultivées au Bénin
}

\author{
Séverin BABATOUNDE ${ }^{1^{*}}$, Madjidou OUMOROU ${ }^{2,3}$, Vincent Isidore TCHABI ${ }^{3}$, \\ Thomas LECOMTE ${ }^{4}$, Marcel HOUINATO ${ }^{1,2}$ et Claude ADANDEDJAN ${ }^{1}$ \\ ${ }^{1}$ Université d'Abomey-Calavi, Faculté des Sciences Agronomiques, Laboratoire de Zootechnie, 01 BP 526 \\ Cotonou, Bénin. \\ ${ }^{2}$ Université d'Abomey-Calavi, Faculté des Sciences Agronomiques, Laboratoire d'Ecologie Appliquée \\ ${ }^{3}$ Université d'Abomey-Calavi, Ecole Polytechnique d'Abomey-Calavi, Laboratoire de Recherche en Biologie \\ Appliquée. \\ ${ }^{4}$ Faculté Universitaire des Sciences Agronomiques, Unité de Zootechnie, Passages des Déportés 2, B 5030 \\ Gembloux, Belgique. \\ *Auteur correspondant ; E-mail : babatoundesev@yahoo.fr
}

\section{RESUME}

Les comportements alimentaires des moutons Djallonké sont étudiés au moyen de 6 espèces de graminées (Andropogon gayanus, Panicum maximum local, Pennisetum purpureum, Panicum maximum var. C1, Panicum maximum var. T58, Brachiaria ruziziensis) et 4 légumineuses fourragères cultivées (Aeschynomene histrix, Stylosanthes fructicosa, Mucuna pruriens var. utilis, Cajanus cajan) récoltées et distribuées aux animaux au stade feuillu ou floraison de leur développement végétatif. $P$. maximum local, $P$. maximum var. $\mathrm{C} 1$ et Pennisetum purpureum sont les mieux ingérés. Les plus faibles valeurs d'ingestion sont enregistrées au stade de 3-4 feuilles par talle pour B. ruziziensis et floraison chez A. gayanus et $P$. maximum var. T58. B. ruziziensis est peu apprécié par rapport à Pennisetum purpureum et aux $P$. maximum. Les animaux préféraient les feuilles du Pennisetum purpureum à celles des $P$. maximum. Entre les $P$. maximum, le local est ingéré préférentiellement par rapport aux espèces sélectionnées. Les légumineuses sont mieux ingérées en début de floraison. L'ingestion de $S$. fructicosa a diminué entre les stades. C. cajan est peu ingérée par les animaux quel que soit son stade. En floraison, les feuilles de $M$. pruriens var. utilis sont préférentiellement ingérées par rapport à celles d'A. histrix et de $S$. fructicosa.

(c) 2010 International Formulae Group. All rights reserved.

Mots clés : Comportements alimentaires, ovins, stades de développement, composition chimique, Bénin.

\section{INTRODUCTION}

L'élevage des petits ruminants est essentiellement pratiqué au Bénin par les petits exploitants et la production des ovins et des caprins est un élément important des systèmes d'exploitation agricole (Reynolds,
1986). Outre la fonction de production, ces animaux jouent un rôle culturel et religieux important (Bamikolé et al., 2001 ; Budisatria et al., 2010). Au Bénin, l'effectif des petits ruminants est estimé en 2007 à 724.000 têtes d'ovins et 1.385.600 têtes de caprins (DE, 
2007). L'attachement des petits exploitants à ce type d'élevage trouve toute sa justification à travers le faible investissement financier, la conduite d'élevage aisée et le cycle de reproduction court des animaux. Il a été démontré que les petits ruminants, facilement monnayables, contribuent pour une part importante aux revenus de ces petits exploitants (Nairan, 1987).

Dans ce milieu, les savanes, les jachères et les champs récoltés sont les sources d'alimentation des animaux tout au long de l'année (Sinsin, 1991). En saison pluvieuse, il $\mathrm{y}$ a une abondance de fourrages de bonne qualité mais ces fourrages déclinent progressivement au fur et à mesure que la saison avance. Afin d'éviter la chute de production pendant la saison déficitaire, il est nécessaire d'envisager une amélioration de la valeur nutritive et une continuité dans la fourniture aux animaux d'une alimentation de bonne qualité. La constitution des réserves fourragères de bonne qualité (foin et ensilage), la complémentation en sous-produits agroindustriels, en sels minéraux et oligoéléments, l'amélioration des pâturages naturels par un débroussaillage et l'introduction de plantes améliorantes ou le développement des cultures fourragères sont des solutions permettant d'atteindre cet objectif. Concernant la production des cultures fourragères, une vaste expérimentation a été réalisée dans le cadre du projet d'Assistance Technique et Scientifique au Département de Production Animale de la Faculté des Sciences Agronmiques de l'Université d'Abomey-Calavi (Bénin). Cette expérimentation avait pour but de comparer la productivité de diverses espèces et variétés de graminées (Andropogon gayanus, Panicum maximum local, $P$. maximum var. $\mathrm{C} 1, P$. maximum var. T58, Brachiaria ruziziensis et Pennisetum purpureum) et de légumineuses (Aeschynomene histrix, Stylosanthes fructicosa, Mucuna pruriens var. utilis et Cajanus cajan) susceptibles d'être cultivées au Bénin. Il s'est avéré que sous le climat tropical humide du Bénin, les meilleures espèces fourragères cultivées peuvent fournir des rendements élevés jusqu'à 11 tonnes de matière sèche par hectare (Buldgen et al., 2001). Connaître la productivité des cultures fourragères est primordiale. Toutefois, elle n'est pas suffisante afin d'établir un plan de rationnement des animaux. Il est donc important que ces fourrages et particulièrement les légumineuses requièrent l'acceptation des animaux et qu'ils soient consommés en quantité suffisante afin de pouvoir satisfaire les productions escomptées. Notre travail a consisté à compléter les précédentes études du projet par une étude approfondie de l'ingestion volontaire et des préférences alimentaires chez des moutons Djallonké nourris avec des graminées et des légumineuses fourragères tropicales cultivées au Bénin.

\section{MATERIEL ET METHODES Milieu d'étude}

Le milieu d'étude est le Bénin situé entre les latitudes $6^{\circ}$ et $12^{\circ}$ nord et les longitudes $1^{\circ}$ et $4^{\circ}$ est. En raison de sa grande extension en latitude, le Bénin offre une succession quasi complète des divers climats intertropicaux. Ces régimes climatiques se différencient par une période sèche et des écarts thermiques qui augmentent tous deux au fur et à mesure que l'on s'écarte du littoral. La température moyenne annuelle est de 27 ${ }^{\circ} \mathrm{C}$ et l'humidité relative moyenne de $70 \%$. La pluviosité varie de 900 à $1.400 \mathrm{~mm}$ par an (INSAE, 2007). Le potentiel agricole du pays est appréciable et la végétation est constituée de savanes, de galeries forestières et de jachères. Les sols sont de nature ferralitique, de texture sableuse, sablo-argileuse ou limoneuse. Ils se caractérisent par de bonnes propriétés physiques, mais leurs qualités chimiques et hydriques sont médiocres (Buldgen et al., 2001).

\section{Préparation du matériel végétal}

Six espèces de graminées fourragères dont trois locales (Andropogon gayanus, Panicum maximum et Pennisetum purpureum) et trois sélectionnées (Brachiaria ruziziensis, Panicum maximum var. $\mathrm{C} 1$ et Panicum maximum var. T58) ont été utilisées. Quatre légumineuses fourragères dont une locale 
(Stylosanthes fructicosa) et trois exotiques (Aeschynomene histrix, Mucuna pruriens var. utilis et Cajanus cajan) ont été utilisées. Les différentes espèces fourragères testées jouaient un rôle important dans les systèmes agro-pastoraux et dans l'alimentation des animaux. Par ailleurs, en ce qui concerne la détermination de l'ingestion volontaire et des préférences alimentaires, il était fondamental de couvrir une large gamme de fourrages cultivés aux caractéristiques morphogénétiques et de croissance distinctes.

Les graminées ont été installées au mois de mai 2007 sur des parcelles de $20 \mathrm{~m}$ x $5 \mathrm{~m}$ avec 5 répétitions par espèce. Avant le démarrage des mesures d'ingestion, chaque parcelle de $100 \mathrm{~m}^{2}$ est subdivisée en 5 petites parcelles de $20 \mathrm{~m}^{2}$, soit au total 25 petites parcelles de $20 \mathrm{~m}^{2}$ par espèce fourragère. Avant le démarrage des mesures d'ingestion, ces parcelles ont été successivement fauchées à un jour d'intervalle, de façon à récolter des fourrages au même stade de développement au cours des mesures d'ingestion volontaire. Dans le but de favoriser la repousse des espèces, les parcelles ont reçu, après chaque coupe, une dose de 25 unités d'azote sous forme d'urée.

Au cours de la période expérimentale, les graminées fourragères ont été fauchées et distribuées aux animaux à différents stades de développement à savoir : 3-4 feuilles ou 4-5 feuilles par talles pour A. gayanus, $B$. ruziziensis, $P$. maximum, $P$. maximum var. $\mathrm{C} 1$ et $P$. maximum var. T $58 ; 8-9$ ou $12-17$ feuilles pour $P$. purpureum; floraison pour toutes les espèces à l'exception du $P$. purpureum qui a été récolté juste avant la floraison. Ainsi, au total 18 fourrages différents de graminées ont été étudiés.

Les fourrages verts des légumineuses ont été récoltés en milieu de croissance végétative et en pleine floraison. Les foins de ces légumineuses ont été récoltés en début de floraison. $C$. cajan était récolté par coupes à $50-60 \mathrm{~cm}$ du sol afin d'éliminer les tiges trop ligneuses et, tout comme pour $M$. pruriens var. utilis, le restant des tiges épaisses était séparé. A. histrix et $S$. fructicosa ont été récoltés et distribués en plante entière, en prenant soin d'éliminer les racines. Les récoltes ont permis d'obtenir 12 fourrages différents pour les légumineuses fourragères étudiées.

\section{Mesures d'ingestion volontaire et des préférences alimentaires}

Pour l'ensemble des mesures réalisées, douze béliers adultes castrés de race Djallonké ont été utilisés. Ces animaux provenaient du centre de sélection ovine de la ferme de FounFoun situé dans la commune de Savè au Centre du Bénin. Chaque mesure d'ingestion a été effectuée à l'aide de 4 moutons choisis aléatoirement ayant un poids vif corporel (PV) moyen de $30 \mathrm{~kg}$. Avant le démarrage des expérimentations, tous les animaux ont été traités au Bérénil contre la trypanosomiase et à l'Ivomec contre les parasites internes et externes. En outre, ils ont eu, en permanence à leur disposition, de l'eau de boisson et des pierres à lécher.

$\mathrm{Au}$ démarrage de chaque mesure d'ingestion, une double pesée des animaux a été faite et les moutons ont été placés dans des cages à métabolisme. Les rations ont été distribuées ad libitum en deux repas espacés de 7 heures soit à $9 \mathrm{~h}$ et à $16 \mathrm{~h}$.

Préalablement à la mesure, la phase d'adaptation des animaux aux cages à métabolisme et aux fourrages a duré 6 à 14 jours. La mesure d'ingestion proprement dite a duré 8 à 10 jours. Chaque jour, une quantité équivalente à $10 \%$ du fourrage distribué a été échantillonnée, stockée dans un sac en plastique et conservée à $-18{ }^{\circ} \mathrm{C}$ au congélateur pour faire les analyses chimiques ultérieures. Tous les matins, les refus alimentaires de chaque mouton sont quantifiés. En outre, 20\% des quantités de fourrages refusées sont échantillonnées par mouton et conservées à $18{ }^{\circ} \mathrm{C}$ au congélateur pour faire les analyses chimiques ultérieures.

Au terme des mesures, les échantillons à $-18{ }^{\circ} \mathrm{C}$ sont décongelés, bien mélangés, échantillonnés et séchés à $60{ }^{\circ} \mathrm{C}$ dans une étuve ventilée jusqu'à l'obtention d'un poids constant. Ces échantillons secs ont ensuite été moulus à $2 \mathrm{~mm}$ (Moulin à marteau) puis à 1 $\mathrm{mm}$ (Cyclotec de Tecator) pour les analyses 
chimiques ultérieures. Au total, trente mesures d'ingestion volontaire ont été ainsi effectuées selon la procédure sus-indiquée. L'ingestion est calculée à partir de la formule suivante :

Nutriment ingéré $\left(\mathrm{g} . \mathrm{j}^{-1}\right)=$ Nutriment distribué $\left(\mathrm{g} . \mathrm{j}^{-1}\right)$ - Nutriment refusé $\left(\mathrm{g} . \mathrm{j}^{-1}\right)$

Par la suite, l'indice de préférence alimentaire (IP) a été déterminé pour les espèces étudiées en utilisant la procédure de Hadjigeorgiou et al. (2003) :

$\mathrm{IP}=\ln$ (ingestion espèce $\mathrm{A} /$ ingestion espèce $\mathrm{B})$, avec :

$\ln =$ logarithme népérien ; Espèce $\mathrm{A}=$ espèce de gauche ; espèce $\mathrm{B}=$ espèce de droite.

Une valeur positive de l'IP indique une préférence pour l'espèce de gauche et une valeur négative de l'IP indique une préférence pour l'espèce de droite.

\section{Détermination de la composition chimique}

La matière sèche (MS, AOAC 967.03), la matière organique (MO, AOAC 923.03) et les matières azotées totales (MAT, AOAC 981.10) ont été déterminées selon les méthodes officielles approuvées par AOAC (1990). Les constituants pariétaux en particulier neutral detergent fiber (NDF), acid detergent fiber (ADF) et la lignine ou acid detergent lignin (ADL) ont été déterminés selon la méthode de Van Soest et al. (1991).

\section{Analyses statistiques}

La statistique descriptive en termes de pourcentage, de moyenne et d'écart-type a été utilisée pour les données d'analyses chimiques et d'ingestion volontaire. Les paramètres chimiques et l'ingestion ont été soumis à une analyse de la variance à deux critères de classification (espèce et stade de développement) en utilisant la procédure PROC GLM du logiciel SAS (Statistical Analysis System V. 8.02 inc., NC, USA).

\section{RESULTATS}

\section{Composition chimique, sélection des fourrages et ingestion volontaire}

La composition chimique des fourrages distribués a été comparée à celle des parties ingérées afin de déceler un éventuel effet de sélection par les moutons. Cet écart a été mesuré pour les matières azotées totales (MAT) et la lignocellulose (ADF). D'une manière générale, l'accroissement de la teneur en lignine (ADL) au fur et à mesure du développement des plantes se traduisait par une augmentation des taux de refus. Les taux de refus ont varié de 24,6 à 40,2\% entre espèces et pouvaient toutefois atteindre $52 \%$ au moment de la floraison chez A. gayanus. Les graminées triées par les animaux avaient des teneurs entre 8,9 à $17,9 \%$ de MAT et 28,6 à 37,3\% d'ADF (Tableau 1) par rapport à la MS. Une influence significative de l'espèce $(\mathrm{P}$ $<0,05)$ et du stade de développement $(\mathrm{P}<$ $0,001)$ a existé sur les teneurs en MAT des fourrages triés. Les teneurs en ADF ont été aussi dépendantes de l'espèce et du stade de développement.

Pour la plupart des espèces, la sélection des MAT et d'ADF par les moutons apparaissait plus importante au moment de la floraison. La teneur élevée en ADL du fourrage dès le départ provoquait une sélection alimentaire par les moutons (Tableau 1). Les ingestions volontaires de MS des graminées par les moutons présentaient des extrêmes assez étendues (45,0 à 71,5 gMS J' $\left.{ }^{1} \mathrm{kp}^{0,75}\right)$. Les valeurs les plus élevées au stade juvénile sont toutes observées chez les variétés de $P$. maximum. Au moment de la floraison, les valeurs extrêmes des ingestions volontaires par les moutons se situaient entre 29,7 et $54 \mathrm{gMS} \mathrm{J}^{-1} \mathrm{kp}^{0,75}$. En partant du stade feuillu à la floraison, l'ingestion des fourrages de A. gayanus et du P. maximum var. T58 par les moutons a chuté de façon importante $(33,7$ et $29,7 \mathrm{gMS} \mathrm{J}^{-1} \mathrm{kp}^{0,75}$ ), alors que celle du $B$. ruziziensis par les moutons a augmenté de 10 gMS J${ }^{-1} \mathrm{kp}^{0,75}$ entre le stade $3-4$ feuilles et 4 5 feuilles par talle. L'ingestion des fourrages de $P$. purpureum par les moutons a diminué très peu entre le stade 12 - 17 feuilles et le début de la floraison. En moyenne, les fourrages les mieux ingérés par les moutons ont été : $P$. maximum et $P$. purpureum pour les espèces locales et $P$. maximum var. $\mathrm{C} 1$ pour les espèces sélectionnées. Parmi les espèces étudiées, $P$. maximum récoltée localement a 
été le fourrage le mieux ingéré par les moutons $\left(62,6 \mathrm{gMS} \mathrm{J}^{-1} \mathrm{kp}^{0,75}\right)$. Les plus faibles valeurs d'ingestion par les moutons sont enregistrées au stade 3-4 feuilles par talle pour $B$. ruziziensis et au moment de la floraison pour $A$. gayanus et $P$. maximum var. T58.

En ce qui concerne les légumineuses, les taux de refus par les moutons ont varié de 28,0 à $40,5 \%$ de la quantité du fourrage distribué (Tableau 2). La sélection en termes de MAT et ADF opérée par les animaux est très hautement influencée par l'espèce et le stade de développement ( $\mathrm{p}<0,0001)$. Une interaction hautement significative $(\mathrm{p}<0,001)$ a existé entre l'espèce et le stade de développement. Les valeurs extrêmes des teneurs en MAT et ADF triées par les moutons oscillaient entre 9,4 et $23,7 \%$ et entre 26,1 et $44,7 \%$ respectivement pour les MAT et l'ADF. Chez M. pruriens var. utilis et $S$. fructicosa, la sélection par les moutons était particulièrement sévère pour les fourrages récoltés au stade végétatif et pour le foin. Les analyses statistiques révélaient une influence très hautement significative $(\mathrm{p}<0,0001) \mathrm{de}$ l'espèce et de son stade de développement sur les paramètres d'ingestion par les moutons. Ainsi, l'ingestion volontaire de matière sèche par les moutons était le critère le plus influencé par l'espèce. Pour l'ensemble des légumineuses étudiées, les valeurs extrêmes d'ingestion volontaire par les moutons se situaient entre 35,8 et $73,3 \mathrm{gMS} \mathrm{J}^{-1} \mathrm{kp}^{0,75}$ au stade végétatif, alors qu'à la floraison, elles

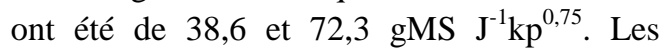
fourrages de légumineuses étudiées étaient pour la plupart mieux ingérés par les moutons en début de floraison, excepté Stylosanthes fructicosa qui a présenté une valeur d'ingestion par les moutons élevée au stade végétatif. Chez cette espèce, l'ingestion par les moutons a diminué très fortement au cours du développement. $C$. cajan était peu appréciée par les animaux quel que soit le stade de développement. En pâture libre, nos observations ont montré que les moutons Djallonké ne s'approchaient pas de $C$. cajan au stade végétatif. Par contre, les moutons broutaient $C$. cajan mais très peu en début floraison.

\section{Préférences alimentaires des moutons Djallonké}

L'observation visuelle des animaux lors de la réalisation des mesures d'ingestion a révélé que les feuilles étaient plus consommées que les tiges. L'indice de préférence (IP) a été calculé en vue d'identifier les espèces qui sont préférentiellement consommées par les moutons. L'indice de préférence alimentaire (IP) calculé sur les MAT ( IP $_{\mathrm{MAT}}$ ) et l'ADF $\left(\mathrm{IP}_{\mathrm{ADF}}\right)$ par les moutons a révélé un rejet de l'A. gayanus par rapport aux autres graminées (Tableau 3). B. ruziziensis a fait également l'objet de rejet par rapport au $P$. purpureum et aux trois variétés de $P$. maximum (local, var. $\mathrm{C} 1$ et var. T 58). En prenant, comme critère d'appréciation de la quantité de feuilles ingérées par les moutons, l' $\mathrm{IP}_{\mathrm{MAT}}$, une réelle préférence des moutons aux feuilles du $P$. purpureum a existé par rapport à celles des $P$. maximum. Nous avons constaté également que, sur le plan des fibres ingérées, les variétés de $P$. maximum sont préférées par les moutons au $P$. purpureum (cf. $\mathrm{IP}_{\mathrm{ADF}}$ ). Entre les $P$. maximum, le local est ingéré préférentiellement par les moutons par rapport aux espèces sélectionnées.

Comparés aux autres légumineuses, les résultats obtenus sur l'indice de préférence alimentaire (IP) calculé sur les MAT (IP MAT $_{\text {) }}$ et l'ADF ( $\left(\mathrm{IP}_{\mathrm{ADF}}\right)$ par les moutons indiquaient un rejet de $C$. cajan (Tableau 4). Les feuilles de $M$. pruriens var. utilis (cf. $\mathrm{IP}_{\mathrm{MAT}}$ ) sont préférentiellement ingérées par les moutons par rapport à celles d'A. histrix et de $S$. fructicosa. Toutefois, il est important de relativiser ce résultat car il s'agissait ici d'une comparaison réalisée sur la moyenne. En réalité, c'est au moment de la floraison que l'ingestion de $M$. pruriens var. utilis par les moutons a été particulièrement élevée (Tableau 4). 
Tableau 1 : Teneur en lignine (ADL, \% MS), taux de refus (\% du fourrage distribué), ingestion $\left(\mathrm{gMS} \mathrm{J}^{-1} \mathrm{~kg}^{0,75}\right.$ ) et sélection animale (distribué et ingéré) en terme de MAT et d'ADF pour les graminées cultivées tropicales $(\%$ de MS) $(n=18)$.

\begin{tabular}{|c|c|c|c|c|c|c|c|c|}
\hline \multirow{2}{*}{ Espèces } & \multirow{2}{*}{ Stade } & \multirow{2}{*}{ ADL } & \multirow{2}{*}{ Refus } & \multirow{2}{*}{ Ingestion } & \multicolumn{2}{|c|}{ MAT } & \multicolumn{2}{|c|}{ ADF } \\
\hline & & & & & \multicolumn{3}{|c|}{ distribué ingéré distribué } & ingéré \\
\hline Andropogon & $3-4$ feuilles & 4,1 & 28,5 & 60,2 & 15,8 & 16,8 & 33,9 & 31,7 \\
\hline \multirow[t]{2}{*}{ gayanus } & $4-5$ feuilles & 5,8 & 39,8 & 57,0 & 8,4 & 9,5 & 36,9 & 33,6 \\
\hline & Floraison & 7,8 & 52,2 & 33,7 & 8,5 & 10,6 & 39,6 & 34,7 \\
\hline Moyenne & & 5,9 & 40,2 & 50,3 & 10,9 & 12,3 & 36,8 & 33,3 \\
\hline Ecart-type & & 1,9 & 11,7 & 14,5 & 4,2 & 3,4 & 2,9 & 1,7 \\
\hline Brachiaria & $3-4$ feuilles & 3,2 & 24,0 & 45,0 & 16,3 & 17,9 & 30,8 & 28,6 \\
\hline \multirow[t]{2}{*}{ ruziziensis } & $4-5$ feuilles & 3,3 & 42,5 & 54,7 & 12,6 & 14,0 & 34,6 & 30,5 \\
\hline & Floraison & 4,7 & 31,0 & 53,7 & 11,1 & 11,8 & 35,3 & 32,8 \\
\hline Moyenne & & 3,7 & 32,5 & 51,1 & 13,3 & 14,5 & 33,6 & 30,6 \\
\hline Ecart-type & & 0,8 & 9,8 & 5,3 & 2,7 & 2,7 & 2,4 & 2,0 \\
\hline Panicum & $3-4$ feuilles & 2,8 & 24,7 & 70,7 & 15,6 & 17,0 & 37,1 & 36,3 \\
\hline \multirow[t]{2}{*}{ maximum } & $4-5$ feuilles & 3,3 & 35,0 & 71,0 & 8,3 & 8,9 & 38,3 & 37,3 \\
\hline & Floraison & 7,0 & 46,0 & 46,2 & 8,4 & 11,6 & 40,9 & 33,4 \\
\hline Moyenne & & 4,4 & 36,2 & 62,6 & 10,8 & 12,1 & 38,8 & 35,6 \\
\hline Ecart-type & & 2,3 & 9,5 & 14,2 & 4,2 & 3,4 & 1,9 & 1,8 \\
\hline Panicum & $3-4$ feuilles & 3,1 & 35,0 & 70,7 & 15,2 & 17,5 & 37,0 & 35,5 \\
\hline \multirow[t]{2}{*}{ maximum var.T58 } & $4-5$ feuilles & 5,3 & 41,7 & 62,3 & 10,9 & 10,9 & 37,3 & 35,8 \\
\hline & Floraison & 6,2 & 38,3 & 29,7 & 10,3 & 12,3 & 42,3 & 38,6 \\
\hline Moyenne & & 5,0 & 38,3 & 54,2 & 11,9 & 13,4 & 39,2 & 36,8 \\
\hline Ecart-type & & 1,6 & 6,2 & 21,7 & 2,7 & 3,0 & 3,0 & 2,0 \\
\hline Panicum & $3-4$ feuilles & 2,7 & 31,8 & 71,5 & 14,1 & 16,7 & 36,0 & 34,2 \\
\hline \multirow[t]{2}{*}{ maximum var.C1 } & $4-5$ feuilles & 4,4 & 36,5 & 58,2 & 10,5 & 12,3 & 36,2 & 34,4 \\
\hline & Floraison & 5,0 & 25,5 & 49,2 & 9,9 & 10,6 & 41,4 & 39,3 \\
\hline Moyenne & & 4,0 & 31,3 & 59,7 & 11,5 & 13,2 & 37,9 & 36,0 \\
\hline Ecart-type & & 1,2 & 6,6 & 11,2 & 2,3 & 2,7 & 3,1 & 2,5 \\
\hline Pennisetum & $8-9$ feuilles & 2,7 & 17,8 & 66,0 & 15,8 & 17,3 & 33,5 & 31,9 \\
\hline \multirow[t]{2}{*}{ purpureum } & $12-\ldots$ & 2,9 & 18,0 & 55,7 & 14,6 & 15,1 & 33,6 & 32,7 \\
\hline & Floraison & 5,1 & 38,0 & 54,0 & 10,6 & 12,1 & 38,1 & 36,1 \\
\hline Moyenne & & 3,6 & 24,6 & 58,6 & 13,7 & 14,8 & 35,1 & 33,6 \\
\hline Ecart-type & & 1,3 & 10,9 & 6,5 & 2,7 & 2,3 & 2,6 & 2,1 \\
\hline Source de variation & $d d l$ & & & & & & & \\
\hline Espèce & 3 & & 0,0001 & 0,0001 & 0,0 & & 0,00 & \\
\hline Stade & 2 & & 0,0001 & 0,0001 & 0,0 & & 0,00 & \\
\hline Espèce $\mathrm{x}$ Stade & 5 & & 0,0001 & 0,0002 & 0,0 & & 0,00 & \\
\hline
\end{tabular}

Tableau 2 : Teneur en lignine (ADL, \% MS), taux de refus (\% du fourrage distribué), ingestion volontaire ( $\mathrm{gMS} \mathrm{J}^{-1} \mathrm{~kg}^{0,75}$ ) et sélection animale (distribué et ingéré) en terme de MAT et d'ADF pour les légumineuses cultivées tropicales $(\%$ de MS) $(n=12)$.

\begin{tabular}{llccccccc}
\hline \multirow{2}{*}{ Espèces } & \multirow{2}{*}{ Stade } & \multirow{2}{*}{ ADL } & \multirow{2}{*}{ Refus } & \multirow{2}{*}{ Ingestion } & \multicolumn{2}{c}{ MAT } & \multicolumn{2}{c}{ ADF } \\
\cline { 7 - 9 } & & & & & & distribu & ingéré distribu & ingéré \\
\hline Aeschynomene & Végétatif & 7,9 & 24,8 & 66,8 & 15,3 & 18,4 & 34,8 & 30,8 \\
histrix & Floraison & 7,5 & 27,3 & 70,6 & 11,8 & 14,0 & 32,2 & 26,3 \\
& Foin & 8,8 & 31,8 & 64,0 & 10,3 & 18,9 & 38,2 & 34,8 \\
Moyenne & & 8,0 & 28,0 & 67,1 & 12,5 & 17,1 & 35,1 & 30,6 \\
Ecart-type & & 0,7 & 3,5 & 3,3 & 2,6 & 2,7 & 3,0 & 4,3 \\
\hline
\end{tabular}




\begin{tabular}{lcccccccc}
\hline \multirow{2}{*}{ Stylosanthes fructicosa } & Végétatif & 11,8 & 35,8 & 73,3 & 13,7 & 15,4 & 31,7 & 27,8 \\
& Floraison & 9,3 & 32,3 & 61,3 & 7,8 & 9,4 & 48,3 & 44,7 \\
Moyenne & Foin & 9,5 & 52,8 & 47,3 & 11,8 & 16,9 & 39,5 & 26,6 \\
Ecart-type & & 10,2 & 40,3 & 60,6 & 11,1 & 13,9 & 39,8 & 33,0 \\
\hline Mucuna & & 1,4 & 11,0 & 13,0 & 3,0 & 4,0 & 8,3 & 10,1 \\
pruriens var. utilis & Végétatif & 7,7 & 39,4 & 54,2 & 17,0 & 23 & 35,4 & 24,4 \\
& Floraison & 6,6 & 11,5 & 72,3 & 18,3 & 18,9 & 28,4 & 26,1 \\
Moyenne & Foin & 9,5 & 52,6 & 47,3 & 17,7 & 23,7 & 38,2 & 23,5 \\
Ecart-type & & 7,9 & 34,5 & 57,9 & 17,7 & 21,9 & 34,0 & 24,7 \\
Cajanus & & 1,5 & 21,0 & 12,9 & 0,6 & 2,6 & 5,0 & 1,3 \\
cajan & Végétatif & 14,2 & 51,8 & 35,8 & 19,6 & 19,0 & 36,1 & 34,1 \\
& Floraison & 13,1 & 38,5 & 38,6 & 14,1 & 14,1 & 32,1 & 30,9 \\
Moyenne & Foin & 12,3 & 31,2 & 30,3 & 20,3 & 21,6 & 32,8 & 30,3 \\
Ecart-type & & 13,2 & 40,5 & 34,9 & 18,0 & 18,2 & 33,7 & 31,8 \\
Source de variation & & 1,0 & 10,4 & 4,2 & 3,4 & 3,8 & 2,1 & 2,0 \\
Espèce & $d d l$ & & & & & & & \\
Stade & 3 & & 0,0001 & 0,0001 & 0,0001 & 0,0001 \\
Espèce x Stade & 2 & & 0,0001 & 0,0001 & 0,0001 & 0,0001 \\
\hline
\end{tabular}

Tableau 3 : L'indice de préférence alimentaire calculé sur les MAT (IPMAT) et l'ADF (IPADF) chez les graminées en seconde année expérimentale quand elles sont comparées deux à deux.

\begin{tabular}{|c|c|c|c|}
\hline $\mathbf{N}^{\circ}$ combinaison & Espèces & $\mathrm{z}_{\text {IP }}$ MAT & $\mathbf{I P}_{\mathrm{ADF}}$ \\
\hline 1 & Andropogon gayanus et Brachiaria ruziziensis & $-0,1571$ & 0,0648 \\
\hline 2 & Andropogon gayanus et Panicum maximum local & $-0,1765$ & $-0,2715$ \\
\hline 3 & Andropogon gayanus et Panicum maximum var. T58 & $-0,1395$ & $-0,1655$ \\
\hline 4 & Andropogon gayanus et Panicum maximum var. $\mathrm{C} 1$ & $-0,2116$ & $-0,2368$ \\
\hline 5 & Andropogon gayanus et Pennetum purpureum & $-0,2971$ & $-0,1533$ \\
\hline 6 & Brachiaria ruziziensis et Panicum maximum local & $-0,0194$ & $-0,3362$ \\
\hline 7 & Brachiaria ruziziensis et Panicum maximum var. T58 & 0,0176 & $-0,2303$ \\
\hline 8 & Brachiaria ruziziensis et Panicum maximum var. $\mathrm{C} 1$ & $-0,0545$ & $-0,3016$ \\
\hline 9 & Brachiaria ruziziensis et Pennetum purpureum & $-0,1400$ & $-0,2180$ \\
\hline 10 & Panicum maximum local et Panicum maximum var. T58 & 0,0370 & 0,1059 \\
\hline 11 & Panicum maximum local et Panicum maximum var. $\mathrm{C} 1$ & $-0,0350$ & 0,0347 \\
\hline 12 & Panicum maximum local et Pennetum purpureum & $-0,1205$ & 0,1182 \\
\hline 13 & Panicum maximum var. T58 et Panicum maximum var. C1 & $-0,0721$ & $-0,0712$ \\
\hline 14 & Panicum maximum var. T58 et Pennetum purpureum & $-0,1576$ & 0,0123 \\
\hline 15 & Panicum maximum var. $\mathrm{C} 1$ et Pennetum purpureum & $-0,0855$ & 0,0835 \\
\hline
\end{tabular}


Tableau 4 : L'indice de préférence alimentaire calculé sur les MAT (IPMAT) et l'ADF (IPADF) chez les légumineuses quand elles sont comparées deux à deux

\begin{tabular}{|c|c|c|c|}
\hline $\begin{array}{l}\mathbf{N}^{\circ} \\
\text { Combinaison }\end{array}$ & Espèces & ${ }^{\mathrm{z}} \mathbf{I P}_{\mathrm{MAT}}$ & $\mathbf{I P}_{\mathrm{ADF}}$ \\
\hline 1 & Aeschynomene histrix et Stylosanthes fructicosa & 0,2805 & 0,0251 \\
\hline 2 & Aeschynomene histrix et Mucuna pruriens var. utilis & $-0,2043$ & 0,3416 \\
\hline 3 & Aeschynomene histrix et Cajanus cajan & 0,5287 & 0,5765 \\
\hline 4 & Stylosanthes fructicosa et Mucuna pruriens var. utilis & $-0,3727$ & 0,3165 \\
\hline 5 & Stylosanthes fructicosa et Cajanus cajan & 0,2482 & 0,5514 \\
\hline 6 & Mucuna pruriens var. utilis et Cajanus cajan & 0,6210 & 0,2349 \\
\hline
\end{tabular}

${ }^{\mathrm{Z}} \mathrm{IP}=\ln$ (ingestion espèce $\mathrm{A} /$ ingestion espèce $\mathrm{B}$ ) ; espèce $\mathrm{A}=$ espèce de gauche ; espèce $\mathrm{B}=$ espèce de droite. Une valeur positive de l'IP indique une préférence pour l'espèce de gauche et une valeur négative de l'IP indique une préférence pour l'espèce de droite.

\section{DISCUSSION}

Nous étudions l'ingestion volontaire et les préférences alimentaires chez des moutons Djallonké nourris avec des graminées et des légumineuses fourragères tropicales cultivées au Bénin. En effet, les repousses des graminées cultivées durant la saison pluvieuse et récoltées au stade de 3 - 4 feuilles par talle fournissent un excellent fourrage car la teneur moyenne de matières azotées totales (MAT) est de $15,5 \%$. Ce qui traduit ainsi la qualité maximale pouvant être obtenue pour ces graminées fourragères. La teneur en lignine des graminées qui est de 3,1\% au stade de 3-4 feuilles par talle, augmente progressivement au cours du développement des plantes. La montaison détermine une proportion plus importante de tiges par rapport aux feuilles des graminées. La montaison double le taux de lignine (ADL) au moment de la floraison $(6,0 \%)$ mais les teneurs de MAT régressent de 15,5 à $9,8 \%$ en moyenne par rapport à la matière sèche (MS) des graminées fourragères. Cette variation de la composition chimique des graminées tropicales au cours du développement est en conformité avec les résultats obtenus par des auteurs comme Minson (1990), Aumont et al. (1995) et Archimède et al. (2009). Les teneurs en MAT et en fibres enregistrées pour $B$. ruziziensis et $P$. maximum var. T58 et $P$. maximum var. C1 concordent assez-bien avec celles mentionnées dans la littérature (Lascano et
Thomas, 1984 ; Reynolds, 1995 ; Roberge et Toutain, 1999). Chez A. gayanus, les MAT sont particulièrement faibles et les teneurs en fibres sont élevées par rapport aux résultats signalés par Lascano et Thomas (1984) et Buldgen et Dieng (1997). Ceci est lié à la gamme variée d'écotypes existant pour $A$. gayanus, induisant une large variabilité de la composition chimique, ainsi qu'au fait que cette espèce se comporte comme une graminée annuelle en climat tropical humide, produisant rapidement des fleurs et des tiges pendant la saison pluvieuse, alors qu'elle est pérenne dans les milieux semi-arides et arides des tropiques (Buldgen et Dieng, 1997; Buldgen et al., 2001).

L'évolution de la composition chimique au cours du développement n'est pas uniforme chez les différentes graminées. Les espèces locales comme $P$. maximum et $A$. gayanus ont des teneurs de MAT diminuant assez rapidement et les fourrages se lignifient beaucoup plus vite au cours du développement. Par contre, ces constituants de la matière organique (MO) évoluent peu pour $P$. purpureum comme l'ont déjà signalé Pieterse et Rethman (2002) et Kozloski et al. (2005). D'ailleurs, Dittberner et Olson (1983), Hendrickson et Briske (1997) et Ramirez et al. (2003) ont également signalé l'importante fluctuation de la composition chimique des espèces locales de graminées au cours du développement. Ici $P$. maximum var. $\mathrm{C} 1$ et $P$. 
maximum var. T58 évoluent assez rapidement, tandis que la composition chimique de $B$. ruziziensis se maintient plus ou moins au cours du développement végétatif. La composition chimique dépend de l'espèce, de la saison, du système cultural et des conditions édaphiques et climatiques (Renard et Compère, 1980 ; César, 1992 ; Compère et al., 1993 ; Herrero et al., 2001).

En dehors des différences de composition chimique, nous notons des différences très nettes entre les espèces de graminées fourragères cultivées du point de vue morphologique. Chez B. ruziziensis et $P$. maximum var. $\mathrm{C} 1$, les feuilles sont rigides et les hampes florales sont fines au stade de 3-4 feuilles par talle. Par contre, les espèces locales $A$. gayanus, $P$. maximum et $P$. purpureum sont des plantes fourragères à tiges et à hampes florales nettement plus épaisses.

L'ingestion volontaire des graminées enregistrée au stade de 3-4 feuilles par talle (64 gMS J-1 $\mathrm{kg}^{0,75}$ en moyenne) concorde bien avec la valeur ( $63 \mathrm{gMS} \mathrm{J}^{-1} \mathrm{~kg}^{0,75}$ ) indiquée par Xandé et al. (1991) pour les graminées fourragères de la zone tropicale humide étudiées aux mêmes stades. L'ingestion des graminées fourragères par les moutons Djallonké varie considérablement. Par exemple, pour un même fourrage comme $P$. maximum var.T58 le mouton ingère $70,7 \mathrm{gMS}$ $\mathrm{J}^{-1} \mathrm{~kg}^{0,75}$ au stade juvénile et seulement 2,4 fois moins $\left(29,7 \mathrm{gMS} \mathrm{J}^{-1} \mathrm{~kg}^{0,75}\right)$ au stade de la floraison. Les teneurs en MAT sont impliquées dans la diminution de l'ingestion volontaire par les animaux des graminées tropicales. Ainsi, comme le signalent des nutritionnistes, il faut un minimum de teneur en MAT ( $8 \%$ de MS) pour assurer un fonctionnement adéquat aux microorganismes du rumen (Milford et Minson, 1966 ; Minson, 1990 ; Coleman et al., 2003). Les teneurs de MAT de 8,3-8,5\% MS aux stades âgés chez A. gayanus et $P$. maximum se situent dans la zone critique où les teneurs en MAT sont limitantes pour le bon fonctionnement du rumen des animaux polygastriques. Eu égard à ces contraintes, les moutons n'ingèrent pas des quantités importantes de fourrages lorsqu'ils sont au stade floraison. Nous avons, toutefois, remarqué que les teneurs élevées en MAT du fourrage ne se traduisent pas nécessairement par des ingestions de MS élevées par les moutons. La teneur en lignine (ADL) augmente en même temps que les taux de refus des fourrages par les moutons. L'augmentation des taux de refus des fourrages par les moutons, au fur et à mesure du développement végétatif des graminées se traduit par une diminution brutale de leurs ingestions volontaires par les animaux. La lignine est le facteur principal de la variation de l'ingestion volontaire de ces graminées fourragères par les moutons. La teneur en ADL peu élevée des graminées au stade de 34 feuilles par talle se traduit par des ingestions volontaires par les moutons élevées (64 gMS $\mathrm{J}^{-1} \mathrm{~kg}^{0,75}$ en moyenne) et un taux de refus des fourrages relativement faible $(27 \%)$. La modification de la composition chimique, en particulier, l'augmentation de la teneur en lignine avec le développement des graminées, se traduit par des taux de refus des fourrages importants (39\%) et des ingestions volontaires par les moutons plus faibles (29 gMS $\mathbf{J}^{-}$ ${ }^{1} \mathrm{~kg}^{0,75}$ ).

Contrairement aux graminées, il n'existe pas de variation importante de la composition chimique des légumineuses au cours du développement végétatif. Par ailleurs, les légumineuses fourragères sont caractérisées par leurs teneurs en MAT toujours plus élevées que celles des graminées fourragères. Pour les fourrages verts de légumineuses, les teneurs en MAT passent de 16,4\% MS au stade végétatif à $13,7 \%$ MS en moyenne au cours de la floraison et le taux de lignine se maintient entre 9,1 et $10,4 \%$ MS. Il apparaît aussi, et ce contrairement aux graminées, que la teneur en lignine des légumineuses ne varie quasiment pas durant le développement végétatif. Il existe cependant des différences de composition chimique et de structure connues depuis longtemps entre les teneurs des lignines des graminées et celles des légumineuses (Jarrige et al., 1995). Les teneurs des lignines de légumineuses 
fourragères ne sont pas plus condensées que celles des graminées fourragères. Toutefois, leur moindre teneur en hydroxyles phénoliques libres explique leur résistance aux solutions alcalines comme le $\mathrm{KOH}$ utilisé lors du dosage de la cellulose brute (Armstrong et al., 1950). Notons que chez $S$. fructicosa d'origine locale, les teneurs en MAT et en fibres (cellulose brute et lignine) évoluent très rapidement au cours du développement végétatif, tout comme pour les graminées fourragères $A$. gayanus, $P$. maximum et $P$. purpureum récoltées localement. La différence de composition chimique observée au niveau des légumineuses fourragères étudiées est aussi liée à leurs différences morphologiques. En effet, les fourrages distribués du $S$. fructicosa et de A. histrix présentent visuellement un rapport feuilles sur tiges plus élevé et ceux du $C$. cajan le plus faible. S. fructicosa et A. histrix sont des plantes herbacées à subligneuses, avec de très petites feuilles, alors que $C$. cajan est une espèce nettement ligneuse et caractérisée par un feuillage très dense. $M$. pruriens var. utilis est, lui aussi, un fourrage à feuillage dense et composé de tiges rampantes qui sont éliminées avant l'affouragement des moutons Djallonké.

Les fourrages de légumineuses présentent toutes, contrairement aux fourrages de graminées, des valeurs de MAT supérieures à la limite critique de $8 \%$ MS. Cependant, de faibles valeurs d'ingestion par les moutons sont observées au stade végétatif (58 $\mathrm{gMS} \mathrm{J}^{-1} \mathrm{~kg}^{0,75}$ en moyenne). La diminution de la teneur en ADL de $13 \mathrm{gkg}^{-1}$ entre le stade végétatif et la floraison des fourrages de légumineuses ne s'accompagne pas, dans les mêmes proportions, d'une augmentation de l'ingestion volontaire par les moutons. Par conséquent, des facteurs antinutritionnels et des substances toxiques sont responsables de la faible ingestion par les moutons des légumineuses fourragères par comparaison à la forte ingestion des graminées fourragères par les moutons. En effet, C. cajan contient des tanins condensés pouvant atteindre jusqu'à $110 \mathrm{~g} \mathrm{~kg}^{-1}$ (Witti et al., 2005). Mieux, à ces composés phénoliques, ajoutons les teneurs élevées en ADL (13,2\% MS) et en matières grasses $(7,1 \%$ MS). Des études réalisées sur la toxicité de Mucuna pruriens révèlent la présence de la L-dopa, des phénols, des tanins et des facteurs antinutritionnels et antitrypsiques (Afolabi et al., 1985 ; Ravindra et Ravindra, 1988 ; Rajaram et Janardhanan, 1991 ; Mary Josephine et Janardhanan, 1992 ; Laurena et al., 1994 ; Dossa et al., 1999), tandis que les évaluations phytochimiques indiquent que Mucuna contient des composés hallucinogéniques apparentés au N,Ndiméthyltryptamine (Lorenzetti et al., 1998). Ainsi, ces constituants peuvent limiter l'ingestion de $C$. cajan et de Mucuna pruriens par les moutons. Au stade végétatif, $S$. fructicosa semble presque dépourvue de tous composés phénoliques ou tanins (Skerman et al., 1988). C'est ce qui explique les ingestions élevées par les moutons au stade feuillu pour cette légumineuse. Des travaux réalisés sur la palatabilité des légumineuses tropicales classent aussi S. fructicosa parmi les espèces à forte ingestion volontaire par les animaux (Peters et al., 2000). Sans la présence des fourrages du C. cajan parmi les divers fourrages distribués aux moutons Djallonké, l'ingestion volontaire des légumineuses augmente de 10 points entre le stade végétatif $\left(58 \mathrm{gMS} \mathrm{J}^{-1} \mathrm{~kg}^{0,75}\right)$ et la floraison (68 gMS J ${ }^{1} \mathrm{~kg}^{0,75}$ ) mais elle n'est pas en moyenne plus élevée que celle des graminées (63 versus 64 gMS $\mathrm{J}^{-1} \mathrm{~kg}^{0,75}$ ). Avec la fenaison, les taux de refus des fourrages par les moutons sont très élevés (jusqu'à $46 \%$ en moyenne) et l'ingestion volontaire par les moutons diminue de $12 \mathrm{gMS} \mathrm{J}^{-1} \mathrm{~kg}^{0,75}$ par rapport aux fourrages verts récoltés au moment de la floraison.

Concernant le comportement sélectif des animaux, nos résultats montrent que les moutons recherchent les mêmes parties des plantes et les mêmes teneurs en nutriments. Ici, le tri des fourrages par les moutons Djallonké est variable suivant le stade de développement végétatif de chaque espèce fourragère. Lorsque les espèces fourragères passent du stade juvénile au stade âgé et que les teneurs en ADL augmentent, les moutons 
consomment plus les feuilles que les tiges. Divers auteurs (Zemmelink, 1980 ; Minson, 1990 ; Faverdin et al., 1997 ; Zemmelink et t'Mannetje, 2002) ont également mentionné le comportement tri des moutons tropicaux qui constitue un paramètre très important de l'ingestion volontaire et de la valeur alimentaire du fourrage une fois devenu très hétérogène et offert en quantité suffisante. Dans le cas des légumineuses le tri de $A$. histrix sur la base de la teneur en MAT par les moutons ne diffère pas significativement $(\mathrm{p}$ > $0,05)$ de celui de $S$. fructicosa sur la base de la teneur en MAT par les moutons. En effet, ces deux légumineuses fourragères renferment très peu de composés phénoliques et les moutons consomment une grande quantité de leurs feuilles pour le simple fait que leur rapport feuilles sur tiges est le plus élevé. Par conséquent, l'espèce fourragère affecte le choix alimentaire des moutons Djallonké. Ainsi, les moutons Djallonké préfèrent les fourrages de $A$. histrix aux autres légumineuses. C'est ce qui explique le fait que les valeurs d'ingestion de A. histrix enregistrées chez les moutons sont les plus élevées et elles restent plus ou moins stables tout au long du développement végétatif de la plante. De même, le stade de développement végétatif des légumineuses influence le choix des animaux. Au moment de la floraison, les moutons préfèrent réellement les feuilles de M. pruriens var. utilis bien qu'elles contiennent des facteurs antinutritionnels. Tout se passe comme si ces facteurs antinutritionnels s'estompent au fur et à mesure du développement végétatif de cette plante. C. cajan apparaît ici comme un fourrage indésirable par les moutons quel que soit son stade de développement végétatif. En effet, la faible ingestibilité de $C$. cajan par les moutons Djallonké s'explique à la fois par ses teneurs en lignine et en matières grasses plus élevées que celles des autres légumineuses. Nous constatons aussi que la forme de présentation de ces légumineuses fourragères (vert ou foin) aux animaux affecte leur ingestion. Enfin, nous constatons également que les feuilles de C. cajan sont plus dures au toucher. Cette caractéristique agit sur l'acceptabilité du fourrage par les animaux car les facteurs physiques et chimiques sont des contraintes pouvant intervenir dans le processus de rassasiement chez l'animal (Faverdin et al., 1997).

\section{Conclusion}

Notre choix ici porte sur l'ingestion volontaire et des préférences alimentaires chez des moutons Djallonké nourris avec des graminées et des légumineuses fourragères tropicales cultivées au Bénin. Les différences morphologiques entre les graminées fourragères se répercutent également sur leur composition chimique. La variabilité des éléments nutritifs, déjà importante entre les espèces fourragères, s'amplifie avec le stade de développement végétatif des plantes. Nos résultats montrent que les moutons Djallonké consomment mieux les graminées locales Panicum maximum et Pennisetum purpureum que les autres graminées sélectionnées Panicum maximum var. $\mathrm{C} 1$, Panicum maximum var. T58 et Brachiaria ruziziensis. Les teneurs en éléments nutritifs des légumineuses Aeschynomene histrix, Stylosanthes fructicosa, Mucuna pruriens var. utilis et Cajanus cajan sont nettement moins variables au cours de leur développement végétatif. Toutefois, excepté $C$. cajan qui présente des caractéristiques nutritionnelles médiocres et est de ce fait mal ingérée par les moutons, les tris des autres légumineuses constituent une source de protéines supplémentaires en fin de saison des pluies lorsque la plupart des graminées ont de faibles valeurs nutritionnelles. Les résultats indiquent par ailleurs que la forme de présentation du fourrage (vert ou foin) influence l'ingestion. L'espèce fourragère affecte le choix alimentaire des moutons Djallonké. Les moutons préfèrent les fourrages de A. histrix aux trois autres légumineuses. De même, le stade de développement des légumineuses influence le choix des animaux. Au moment de la floraison des légumineuses, les moutons préfèrent réellement les feuilles de $M$. pruriens var. utilis. Etant donné qu'une 
grande partie des légumineuses est susceptible de contenir des quantités importantes de facteurs antinutritionnels responsables notamment des faibles valeurs d'ingestion volontaire par les moutons, les investigations doivent se poursuivre en dosant en quantité et en qualité ces molécules et plus particulièrement les tanins associés aux lignines.

\section{REMERCIEMENTS}

Les auteurs remercient la coopération technique belge (CTB) et le projet belgobéninois (FSA5/CIUF/UAC), pour avoir financé ce programme de recherche sur l'ingestion et les préférences alimentaires des graminées et des légumineuses fourragères cultivées au Bénin.

\section{REFERENCES}

Afolabi OA, Oshuntogun BA, Aadewusi SR, Fapojuwo OO, Ayorinde FO, Grisson FE, Oke OL. 1985. Premimynary Nutritional and chemical evaluation of Raw seeds from Mucuna Solanei: an underutilized Foods source. J. Agric-Food Chem., 33: 122-124.

Akondé TP., Leihnez DE., Kûlne F., Steinmûller N. 1997. Allez cropping on an ultisol in subhumid Benin 3. Nutrient budget of maize, cassava and trees. Agrofor. Syst., 37 : $213-216$.

AOAC (Association of Official Analytical Chemists). 1990. Official Methods of Analysis $\left(15^{\text {th }}\right.$ edn). INC, Va: USA.

Archimède H, Sauvant D, Assoumaya C, Fanchone A, Boval M. 2009. Fourrages tropicaux: valeur alimentaire comparée aux fourrages tempérés et évaluation au pâturage. Renc. Rech. Ruminants, 16: 1724.

Armstrong DG, Cook H, Thomas B. 1950. The lignin and cellulose contents of certain grassland species at different stages of growth. J. Agric. Sci., 40: 93-99.

Aumont G, Caudron I, Saminadin G, Xandé, A. 1995. Source of variation in nutritive values of tropical forages from the
Caribbean. Anim. Feed. Sci. Technol., 51: $1-13$.

Bamikole MA, Ezenwa I, Akinsoyinu AO, Arigbede MO, Babayemi OJ. 2001. Performance of West African dwarf goats fed Guinea grass - Verano stylo mixture, N-fertilized and unfertilized Guinea grass. Small Rumin Res., 39: 145 -152.

Budisatria IGS, Udo HMJ, Eilers CHAM, Baliarti E, Van der Zijpp AJ. 2010. Preferences for sheep or goats in Indonesia. Small Rumin. Res., 88: 16-22.

Buldgen A, Michiels B, Adjolohoun S, Babatoundé S, Adandédjan CC. 2001. Production and nutritive value of grasses cultivated in the coastal area of Benin. Tropical Grasslands, 35: 43- 47.

Buldgen A, Dieng A. 1997. Andropogon gayanus var. bisquamulatus. Une Culture Fourragère pour les Régions Tropicales. Les Presses Agronomiques de Gembloux: Gembloux, Belgium.

César J. 1992. Etude de la production biologique des savanes de Côte-d'Ivoire et de son utilisation pour l'homme, biomasse, valeur pastorale et production fourragère. Thèse de doctorat, Paris VI, p.642.

Coleman SW, Hart SP, Sahlu T. 2003. Relationships among forage chemistry, rumination and retention time with intake and digestibility of hay by goats. Small Ruminant Research, 50: 129-140.

Compère R, Hellemans $\mathrm{P}$, Tommassini $\mathrm{M}$, Buldgen A. 1993. Les savanes du ranch de l'Adélé (Togo). L'exploitation actuelle des ressources fourragères et proposition pour une gestion plus rationnelle. Bull. Rech. Agron. Gembloux 28 (1): 29-50.

DE (Direction de l'Elevage). 2007. Programme de développement des productions animales pour les cinq prochaines années (2007-2012). Rapport de la Direction de l'Elevage, MAEP/Cotonou/Bénin, p. 159.

Dittberner PL, Olson ML. 1983. The plant Information Network (PIN) Data Base: Colorado, Montana, North Dakota, Utah, and Wyoming, FWS/OBS-83-86. US 
Department of Interior, Fish and Wildlife Service, Washington, DC, p. 786.

Dossa CS, Mensah GA, Dossa AD, Adoun C. 1999. Influence de divers traitements physico-chimiques de graines de Mucuna pruriens sur leur composition chimique en nutriments. Tropicultura, 3(16-17): 141-146.

Faverdin P, Agabriel J, Bocquier F, Ingrand S. 1997. Maximiser l'ingestion de fourrages par les ruminants : maîtrise des facteurs liés aux animaux et à leur conduite. Renc. Rech. Ruminants, 4: 65-74.

Hadjigeorgiou IE, Gordon IJ, Milne JA. 2001. The intake and digestion of a range of temperate forages by sheep and fibreproduction goats. Small Rumin. Res., 39: 167-179.

Hendrickson JR, Briske DD. 1997. Axillary bud banks of two semiarid perennial grasses : occurrence, longevity and contribution to population persistence. Oecologia, 110: 584-591.

Herrero M, do Vall CB, Hughes NRG, Sabatel V de O, Jessop NS. 2001. Measurement of physical strength and their relationship to chemical composition of four species of Brachiaria. Anim. Feed. Sci. Technol, 92: $149-158$.

INSAE. 2007. Ann. Insti Nat. Stat. Ana Eco, Cot. pp 23-35

Jarrige R, Dulphy JP, Faverdin P, Baumont R, Demarquilly C. $1995 . \quad$ Activité d'ingestion et de rumination. In Nutrition des ruminants domestiques : ingestion et digestion, Jarrige $\mathrm{R}$, Ruckebusch $\mathrm{Y}$, Demarquilly C, Farce MH, Journet M (eds) ; 123-181.

Kozloski GV, Perottoni J, Ciocca MLS, Rocha JBT, Raiser AG, Sanchez LMB. 2003. Potentiel nutritional assessment of dwarf elephant grass (Pennisetum purpureum Schum. Cv. Mott) by chemical composition, digestion and net portal flux of oxygen in cattle. Anim. Feed. Sci. Technol., 104: 29-40.

Lascano C, Thomas D. 1984. Forage quality and animal selection of Arachis pintö̈ in association with tropical grasses in the eastern plains of Colombia. Grass and Forage Sci., 43: 433-439.

Laurena AC, Revilleza MJR, Mendoza EMT. 1994. Polyphenols, Phytates Cyanogenetic, Glycosids and Trypsin inhibitor activity of several Phillipine indigenous food legumes. J. Food Composition and Analysis, 7: 194-202.

Lorenzetti FS, Maclsaac JT, Arnason DVC, Awang R, Buckles D. 1998. The phytochemistry, Toxicology and food potential of Velvetbean. In Contributing to sustainable Agriculture, Buckles D, Eteka E, Osiname O, Galiba M, Galiano $\mathrm{G}$ (eds). Cover Crops in West Africa; 6784.

Mary Josephine R, Janardhanan K. 1992. Studies on chemical composition and anti nutritional Factors in Germplasm Seed Materials of the tribal pulse, Mucuna pruriens (L) DC. Food Chemistry, 43: 1318.

Médénou C, Soulé M. 1988. Notes techniques sur l'élevage en RPB. $\mathrm{N}^{\circ} 411$, MFEEP/Cotonou/Bénin, p. 53.

Milford R, Minson DJ. 1966. Intake of tropical pasture species. Proceedings of the IX International Grasslands Congress 1: 815-822.

Minson DJ. 1990. The chemical composition and nutritive value of tropical grasses. In Tropical Grasses, Skerman PJ, Riveros F (eds). FAO plant production and protection series, 23, Food and Agriculture Organization.

Nairant T. 1987. Le rôle des petits ruminants dans le développement. Courrier AfriqueCaraïbes Pacifique, 104: 17-23.

Peters M, Tarawali SA, Schultze-Kraft R. 2000. Relative palatability and seasonal agronomic performance of species mixtures in subhumid West-Africa. Experimental Agriculture, 36: 353-368.

Pieterse PA, Rethman NFG. 2002. The influence of nitrogen fertilisation and soil $\mathrm{pH}$ on the dry matter yield and forage quality of Pennisetum purpureum and $P$. purpureum $x \quad P$. glaucum hybrids. Tropical Grasslands, 36: 83-89. 
Rajaram N, Janardhanan K. 1991. The biochemical composition and nutritional potential of the tribal pulse, Mucuna gigantea (Willd.) DC. Plants Foods for Human Nutrition, 44: 45-51

Ramirez RG, Haenlein GFW, Garcia-Castillo CG, Núñez-González MA. 2003. Protein, lignin and mineral contents and in situ dry matter digestibility of native Mexican grasses consumed by range goats. Small Rumin. Res., 52(3): 261 - 269.

Ravindran V, Ravindran G. 1988. Nutritional and anti-nutritional characteristics of Mucuna (Mucuna utilis) bean seeds. J. Sci. Food. Agric. 46: 71-79.

Renard JF, Compère R. 1980. Le potentiel pastoral des savanes guinéennes au Togo. Bull. Rech. Agron. Gembloux, 14(3-4): 253-266.

Reynolds L. 1986. Elevage des petits ruminants: situation actuelle et possibilité de développement par l'amélioration de l'alimentation. Bull. CIPEA, 25: 13-17.

Reynolds SG. 1995. Pasture - Cattle Coconut systems. FAO : Rome, Italy. p. 664.

Roberge G, Toutain B. 1999. Cultures Fourragères Tropicales. CIRAD: Montpellier, France.

Sinsin B. 1991. Exploitation des pâturages dans un système traditionnel d'élevage bovin dans le périmètre Nikki-Kalalé au Bénin. IV $^{\text {th }}$ Congrès International des Terres de Parcours, Montpellier, France, p. 660-662.
Skerman PJ, Riveros F. 1988. Tropical forage legumes. FAO plant production and protection series 2. Food and Agriculture Organization (FAO), Rome, Italy, p. 692.

Van Soest PJ, Robertson, JB, Lewis BA. 1991. Methods for dietary fiber, neutraldetergent fiber and-starch polysaccharides in relation to animal nutrition. J. Dairy Sci., 74: 3583-3597.

Xandé A, Aumont G, Caudron I. 1991. Tables des valeurs alimentaires des fourrages tropicaux de la zone Caraïbe et de la Réunion. Pointe à Pitre, CRZ, Guadeloupe.

Witti DMSS, Abdalla AL, Bueno ICS, Silva Filho JC, Costa C, Bueno MS, Nozella EF, Longo C, Vieira EQ, Cabral Filho SLS, Godoy PB, Mueller-Harvey I. 2005. Do all tannins have similar nutritional effects ? A comparison of three Brazilian fodder legumes. Anim. Feed Sci. Technol., 119: 345-361.

Zemmelink G. 1980. Effect of selective consumption on voluntary intake and digestion of tropical forages. Agric. Res. Rep., 896: 100.

Zemmelinke G, t'Mannetje L. 2002. Value for animal production (VAP) : a new criterion for tropical forage evaluation. Anim. Feed. Sci. Technol., 96: 31-42. 